\title{
DETECTION OF A 60-LONG DWARF GALAXY DEBRIS STREAM
}

\author{
C. J. Grillmair \\ Spitzer Science Center, 1200 East California Boulevard, Pasadena, CA 91125; carl@ipac.caltech.edu \\ Received 2006 April 20; accepted 2006 May 16; published 2006 June 23
}

\begin{abstract}
We report on a $60^{\circ}$-long stream of stars, extending from Ursa Major to Sextans, in the Sloan Digital Sky Survey. The stream is approximately $2^{\circ}$ wide and is clearly distinct from the northern tidal arm of the Sagittarius dwarf galaxy. The apparent width of the stream indicates a progenitor with a size and mass similar to that of a dwarf galaxy. The stream is about $21 \mathrm{kpc}$ distant and appears to be oriented almost perpendicular to our line of sight. The visible portion of the stream does not pass near any known dwarf galaxies, although we cannot rule out that the stream may form the inner part of a known dwarf galaxy's orbit. The most likely explanation is that the stream constitutes the remains of a dwarf galaxy that has been completely disrupted at some point in the past. We also briefly report on the discovery of a diminutive Galactic satellite that lies near the projected path of the new stream but is unlikely to be related to it.
\end{abstract}

Subject headings: Galaxy: halo — Galaxy: structure — globular clusters: general

\section{INTRODUCTION}

The Sloan Digital Sky Survey (SDSS) continues to be a remarkable resource for studies of Galactic structure and the Local Group. In addition to the large-scale features attributed to past galaxy accretion events (Yanny et al. 2003; Majewski et al. 2003; Rocha-Pinto et al. 2004), SDSS data were used to detect the remarkably strong tidal tails of Palomar 5 (Odenkirchen et al. 2001; Rockosi et al. 2002; Odenkirchen et al. 2003; Grillmair \& Dionatos 2006a). Most recently, Willman et al. (2005), Zucker et al. (2006), and Belokurov et al. (2006b) used SDSS data to discover several new dwarf satellites of the Milky Way.

Substantial tidal streams have now been found that are associated with at least two of the eight globular clusters in the SDSS area: Pal 5 (Odenkirchen et al. 2003; Grillmair \& Dionatos 2006a) and NGC 5466 (Belokurov et al. 2006a; Grillmair \& Johnson 2006). Most recently, Grillmair \& Dionatos (2006b) discovered a $63^{\circ}$ cold stellar stream without an obvious progenitor. Although the parent body may be one of the $\approx 140$ globular clusters situated outside the SDSS area, it is equally possible that the stream is all that remains of a globular cluster that was disrupted long ago.

In this Letter we analyze a broad, $60^{\circ}$-long stream, independently detected in SDSS data by Belokurov et al. (2006b). We briefly describe our analysis in $\S 2$. We discuss the detection of the stellar stream in $\S 3$, make some initial distance estimates in $\S 3.1$, and attempt to identify a progenitor in $\S 3.2$. We make concluding remarks in $\S 4$.

\section{DATA ANALYSIS}

Data comprising ugriz photometry for $5.3 \times 10^{7}$ stars in the region $124^{\circ}<\alpha<251^{\circ}$ and $-1^{\circ}<\delta<65^{\circ}$ were extracted from the SDSS database using the SDSS CasJobs query system. The data were analyzed using the matched filter technique employed by Grillmair \& Johnson (2006) and Grillmair \& Dionatos (2006a, 2006b) and described in detail by Rockosi et al. (2002). This technique is made necessary by the fact that, over the magnitude range and over the region of sky we are considering, the foreground disk stars outnumber the more distant stars in the Galactic halo by 3 orders of magnitude. Applied in the color-magnitude domain, the matched filter is a means by which we can optimally differentiate between two populations, provided we know the color-magnitude distribution of each.

We used the SDSS photometry to create a color-magnitude density or Hess diagram for both stars of interest and for the contaminating foreground population. Dividing the former by the latter, we generated an array of relative weights that constitutes an optimal color-magnitude filter. Using this filter, every star in the survey can be assigned a weight or probability of association with a particular color-magnitude sequence. Having used observed data to generate it, the filter implicitly includes the effects of photometric uncertainties.

To generate the filters, we used the SDSS-observed colormagnitude distributions for stars in each of the eight globular clusters in the SDSS Data Release 4 (DR4) area (NGC 2419, Pal 3, NGC 5272, NGC 5466, Pal 5, NGC 6205, NGC 7078, and NGC 7089). A single Hess diagram for field stars was generated using $1.2 \times 10^{7}$ stars spread over $\sim 2200 \mathrm{deg}^{2}$ of DR4. We applied each of the eight optimal filters in turn to the entire survey area, and the weighted star counts were summed by location on the sky to produce two-dimensional probability maps.

We used all stars with $15<g<22.5$. We dereddended the SDSS photometry as a function of position on the sky using the DIRBE/IRAS dust maps of Schlegel et al. (1998). We optimally filtered the $g-u, g-r, g-i$, and $g-z$ star counts independently and then co-added the resulting weight images. In Figure 1 we show the final, combined, filtered star count distribution, using a filter matched to the color-magnitude distribution of stars in M13. The image has been smoothed with a Gaussian kernel with $\sigma=0.2$. A low-order, polynomial surface has been subtracted from the image to approximately remove large-scale gradients due to the Galactic disk and bulge.

\section{DISCUSSION}

Quite obvious in Figure 1 are two broad streams, one running east to west across the center of the field, and the other running more nearly north and south. The stronger, east-west stream is part of the well-known northern tidal tail of the Sagittarius dwarf galaxy (Majewski et al. 2003; Martinez-Delgado et al. 2004). The north-south stream is clearly distinct from the Sagittarius stream (intersecting at an angle of almost $45^{\circ}$ on the sky) and runs from (R.A., decl.) $=\left(163^{\circ},-1^{\circ}\right)$ to approxi- 


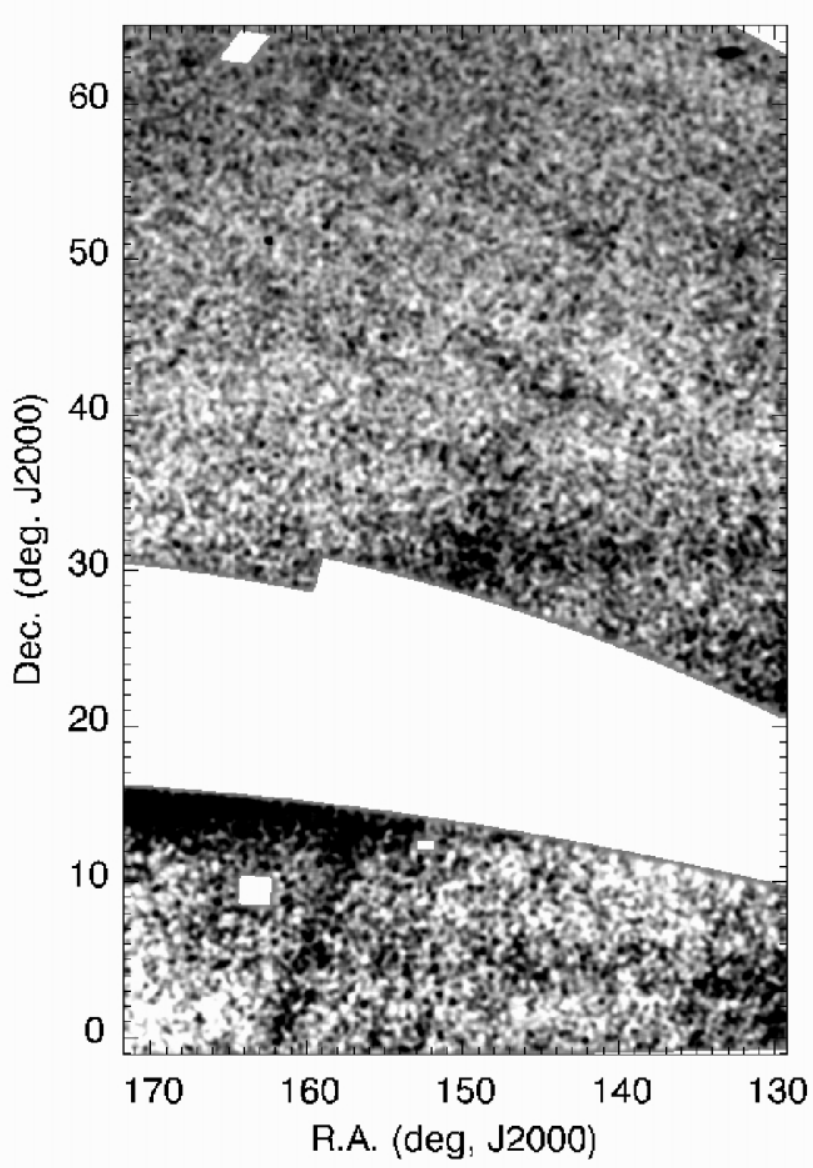

FIG. 1.-Smoothed, summed weight image of the SDSS field after subtraction of a low-order polynomial surface fit. Darker areas indicate higher surface densities. The weight image has been smoothed with a Gaussian kernel with $\sigma=0.2$. The white areas are either data missing from DR4 or clusters or bright stars that have been masked out prior to analysis. The northern tidal arm of the Sagittarius dwarf galaxy runs roughly east-west above and below the gap in DR4. The new stream runs from (R.A., decl.) $=\left(163^{\circ},-1^{\circ}\right)$ to approximately (R.A., decl.) $=\left(141^{\circ}, 52^{\circ}\right)$. At the extreme upper right is a new Milky Way satellite that is considered in detail by C. J. Grillmair (2006, in preparation).

mately $($ R.A., decl. $)=\left(141^{\circ}, 52^{\circ}\right)$. On the sky, the stream runs in a $60^{\circ}$, nearly great circle path from Ursa Major in the north to Sextans in the south. The stream is distinct from the lowlatitude halo stream found by Yanny et al. (2003), which lies some $30^{\circ}$ to the east of the present stream. The stream is also visible in Figure 1 of Belokurov et al. (2006b).

The stream was found using a filter that is matched to the color-magnitude distribution and luminosity function of stars in the globular cluster M13, although shifted faintward by 2.1 mag. We used M13's color-magnitude locus primarily because, owing to M13's relative proximity, we have a better measure of its luminosity function and the effects of SDSS completeness.

The stream is not a product of our dereddening procedure. Figure 2 shows the portion of the reddening map of Schlegel et al. (1998) covering the region of interest. There appears to be no correlation between the new stream and the applied reddening corrections. The maximum values of $E(B-V)$ are $\approx 0.05$, with typical values of 0.03 in the southern region of the stream and 0.01 in the northern. There are diminutions here and there in the stream that could perhaps be attributed to regions of higher reddening in Figure 2, but there is certainly

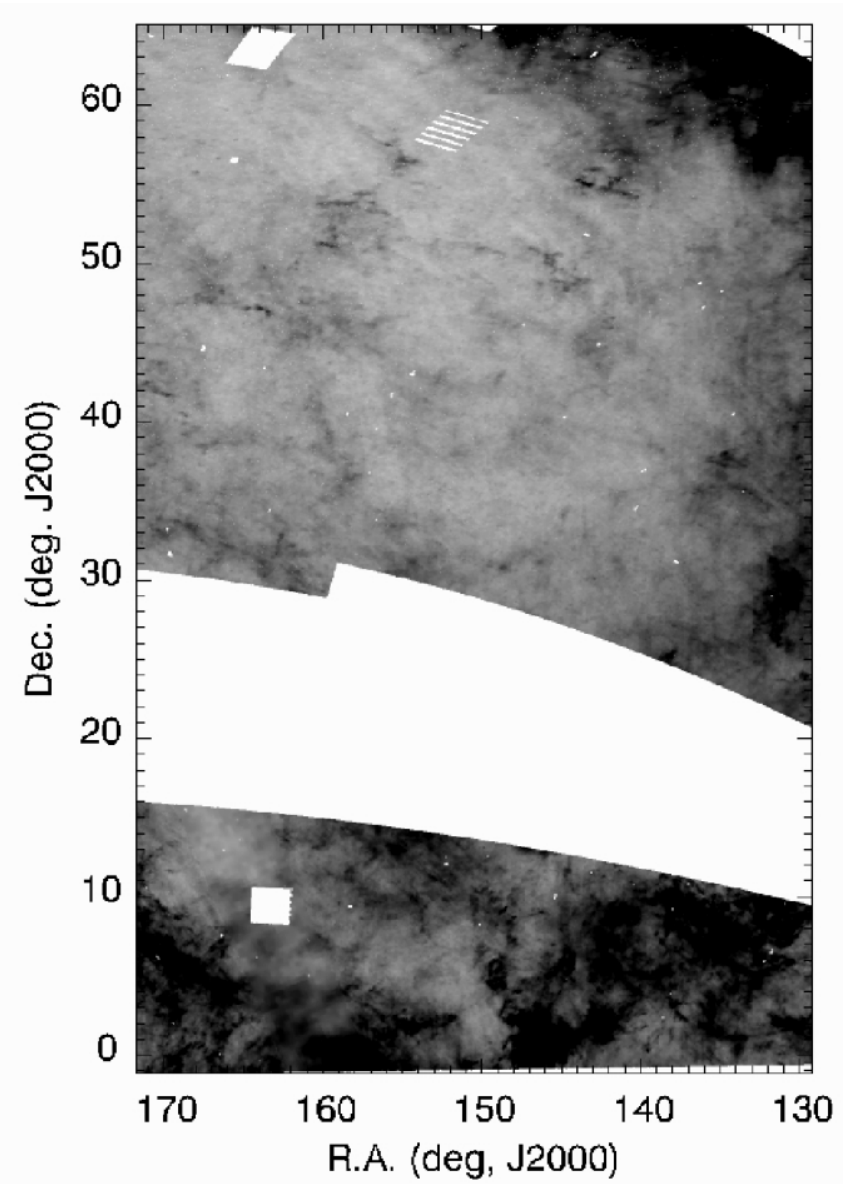

FIG. 2.-Distribution of $E(B-V)$ over the field shown in Fig. 1. Darker areas indicate higher color excesses. Typical values of the color excess range from 0.01 along the northern part of the new stream to 0.03 along the southern part.

no long trough in the reddening map that could be held to account for the entire stream.

We have also run our optimum filter against the SDSS DR4 galaxy catalog to investigate whether the stream could be due to confusion with faint galaxies. Although there are occasional concentrations of galaxies along the stream's trajectory, there is no indication of a continuous north-south feature as is apparent in Figure 1. We conclude that faint galaxies also cannot be held to account for the stream.

At its southern end, the stream is truncated by the limits of the available data. The apparent surface density of the stream is highest at this point, and we are fairly confident that larger surveys will be able to extend the current stream substantially to the south. On the northern end, the stream becomes indiscernible beyond $\delta=51^{\circ}$. We have attempted to trace the stream in the portion of the SDSS extending from $52^{\circ}<\delta<65^{\circ}$ by shifting the filter to both brighter and fainter magnitudes, but to no avail.

We conclude that we are seeing either (1) a substantial local drop in volume density at the northern end of the stream (due perhaps to an episodic stripping process or a higher velocity portion of an eccentric orbit), (2) a significant change in the color-magnitude distribution of stars along the stream, or (3) the physical end of the stream. We consider the second option to be the least likely of the three possibilities, although it is not inconceivable that distinct stellar populations could have been 
pulled from the progenitor in sequence. Arguments for or against the first and third options will have to await the acquisition of survey data covering a larger field.

Sampling by eye at several representative points, the stream appears to be about $2^{\circ}$ wide on average. This is significantly broader than the globular cluster streams found by Odenkirchen et al. (2003), Grillmair \& Johnson (2006), and Grillmair \& Dionatos (2006a, (2006b). On the other hand, it is similar to the western portion of the Sagittarius stream visible in Figure $1 ; 2^{\circ}$ corresponds to about $700 \mathrm{pc}$ at our estimated distance to the stream (see below). If the stream is circular in cross section, then in a logarithmic potential with $v_{c}=220 \mathrm{~km} \mathrm{~s}^{-1}$, a radial width of this magnitude would correspond to $\Delta E>20 \mathrm{~km} \mathrm{~s}^{-1}$. This is considerably larger than the expected random velocities of stars weakly stripped from globular clusters and implies a much larger progenitor mass. Of course, with the stream passing high over the outer regions of the disk $\left(165^{\circ}<l<252^{\circ}, 44^{\circ}<b<50^{\circ}\right)$, we cannot currently say how broad the stream is along a radial from the Galactic center. However, $700 \mathrm{pc}$ remains much larger than the tidal diameters of globular clusters, and we conclude that the progenitor is most likely to be an extant or disrupted dwarf galaxy.

Integrating the background-subtracted, weighted star counts along the stream over a width of $\approx 2^{\circ}$, we find that the total number of stars in the discernible stream down to $g=22.5$ is $1250 \pm 200$. The surface density of stars in the southern portion of the stream $\left(-1^{\circ}<\delta<14^{\circ}\right)$ is roughly twice that of the northernmost section $\left(39^{\circ}<\delta<52^{\circ}\right)$. The highest surface densities

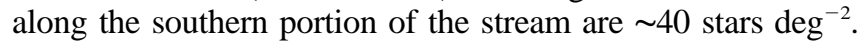

\subsection{Distance to the Stream}

The power of the matched filter resides primarily at the mainsequence turnoff and below, where the stellar luminosity function increases rapidly and the bulk of the foreground contaminants lie well to the red. If our filter is globally matching the colormagnitude distribution of the stream population, then we can use the filter response to estimate distances by main-sequence fitting (e.g., Grillmair \& Dionatos 2006b). In Figure 3 we show the color-magnitude distribution for the stream stars, extracted by generating a Hess diagram of stars in a $11 \mathrm{deg}^{2}$ area covering the southern portion of the stream, and subtracting a similar field star distribution sampled over $28 \mathrm{deg}^{2}$ to the east and west of the stream. A clear signature of the subgiant branch and the main-sequence turnoff is evident in the stream population. Moreover, the subgiant and turnoff region of the distribution match the dereddened, shifted $g, g-i$ red giant branch/main-sequence locus of M13 very well.

Varying the magnitude shift applied to M13's main-sequence locus from 1.5 to $3.0 \mathrm{mag}$, we measured the foregroundsubtracted, mean surface density of stream stars in the regions $-1^{\circ}<\delta<14^{\circ}, 29^{\circ}<\delta<39^{\circ}$, and $39^{\circ}<\delta<52^{\circ}$. To avoid potential problems related to a difference in age between M13 and the stream stars, we used only the portion of the filter with $19.5<g<22.5$, where the bright cutoff is 0.8 mag below M13's main-sequence turnoff. This reduces the stream contrast significantly but still provides sufficient signal to identify the optimum magnitude shift.

Fitting Gaussians to the mean surface densities as a function of magnitude shift (e.g., Grillmair \& Dionatos 2006b), we find that the highest contrasts occur for shifts of 2.1, 2.4, and $2.15 \pm 0.1 \mathrm{mag}$ for the three stream segments identified above. This puts the stream roughly perpendicular to our line of sight

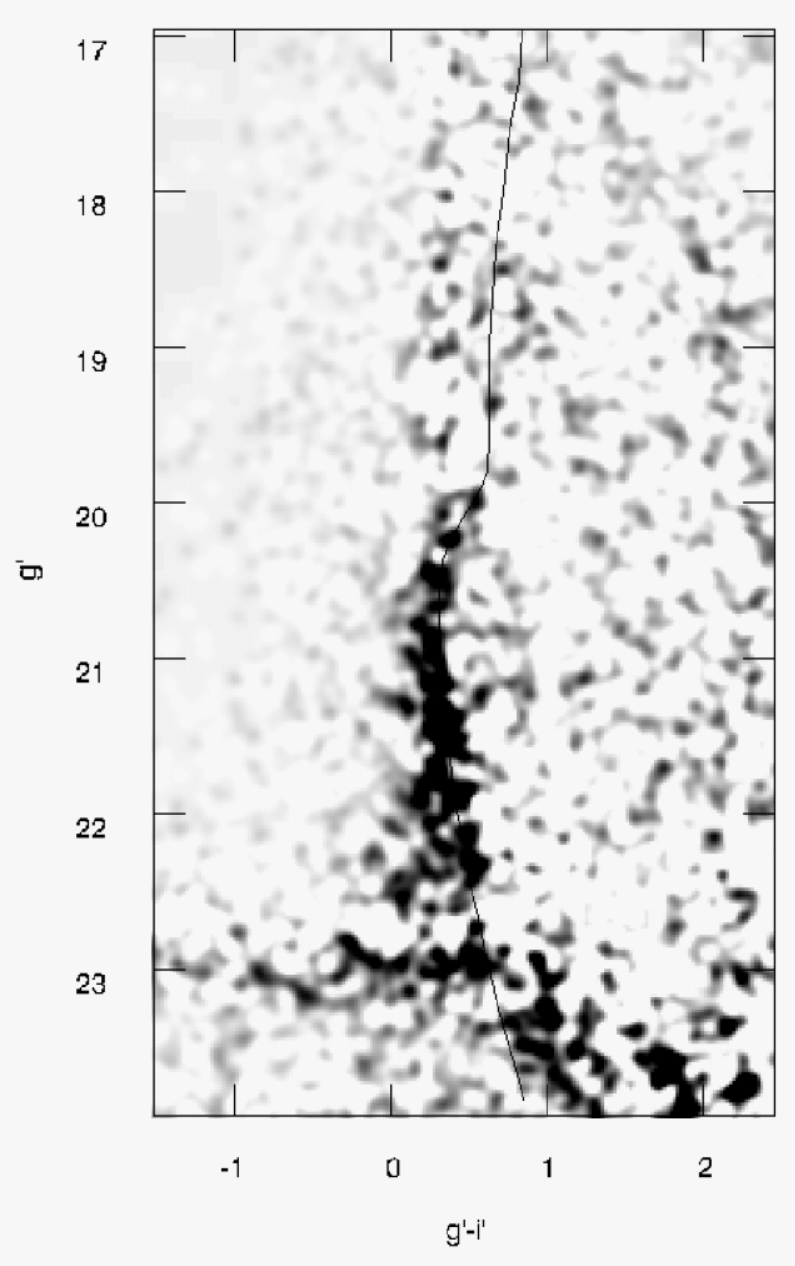

FIG. 3.-Dereddened, background-subtracted, color-magnitude distribution of stream stars. The stream stars include all stars situated in an $11 \mathrm{deg}^{2}$ area covering the southern portion of the stream in Fig. 1. The distribution of field stars was computed from all stars in a $28 \mathrm{deg}^{2}$ area bracketing the stream to the east and west. A subgiant branch and main-sequence turnoff are clearly distinguishable. The solid line shows the dereddened locus of giant branch and main-sequence stars as measured in DR4 for M13, shifted faintward by $2.1 \mathrm{mag}$.

at an average distance 2.78 times that of M13. Adopting a distance to M13 of $7.7 \mathrm{kpc}$, we arrive at a mean Sun-stream distance of $21.4 \pm 1 \mathrm{kpc}$, where the uncertainty reflects only random measurement errors.

The measurement of relative distances in this manner rests on the assumption that the color-magnitude distribution of stream stars is uniform and that different parts of the stream will respond to magnitude shifts in the filter in the same way. Of course, this may not be true if different subpopulations of the parent galaxy were removed at different times. Our relative distance estimates may also be subject to variations in SDSS sensitivity and completeness at faint magnitudes, although over the scales with which we are dealing, it seems reasonable to suppose that the such variations will have largely averaged out.

\subsection{The Stream Progenitor}

The stream does not pass near any of the known dwarf galaxies in the Local Group. While Leo I and Leo A lie close to the stream in projection, their respective distances of $254 \pm 19 \mathrm{kpc}$ (Bellazzini et al. 2004) and $800 \pm 40 \mathrm{kpc}$ (Dolphin et al. 2002) 
put them well beyond the $21 \mathrm{kpc}$ distance that we determined above. The Sextans dwarf is situated about $7^{\circ}$ west of the southern end of the stream. However, at its distance of $86 \pm 6 \mathrm{kpc}$ (Mateo et al. 1995), it too is too far away to be a likely progenitor. At this distance, its main-sequence turnoff would be well below the completeness limit of the SDSS data, which is clearly inconsistent with Figure 3.

Although we are obviously limited by the lack of velocity information, for a given model of the Galactic potential, the progenitor's orbit is actually fairly well constrained by the observed distance and orientation of the stream. Using the model of Allen \& Santillan (1991, which includes a disk, bulge, and spherical halo and which Grillmair \& Johnson 2006 and Grillmair \& Dionatos 2006a found to work reasonably well for NGC 5466 and Pal 5), we use a least-squares method to fit both the orientation on the sky and the distance measurements in § 3.1. In addition to a number of normal points lying along the center line of the stream, we chose as a velocity fiducial point a position at the northern tip of the stream at (R.A., decl.) $=\left(141.2,51^{\circ} .2\right)$.

If we allow the proper motions to be free-ranging and uninteresting parameters, we find that the $95 \%$ confidence interval in $\chi^{2}$ predicts $-35<v_{\mathrm{LSR}}<+57 \mathrm{~km} \mathrm{~s}^{-1}$ at the fiducial point. The corresponding ranges in perigalactic and apogalactic radii are $9.1 \mathrm{kpc}<R_{p}<9.3 \mathrm{kpc}$ and $28 \mathrm{kpc}<R_{a}<32 \mathrm{kpc}$. Of course, these ranges do not take into account uncertainties in the absolute distance of the stream (which depends on the uncertainty in M13's distance) or in the validity of the Allen \& Santillan (1991) Galactic model. However, they do suggest a fairly compact orbit with moderate eccentricity.

Integrating orbits for parameter sets spanning the range above, we find that there are another five known dwarf galaxies (NGC 185, the Aquarius dwarf galaxy, NGC 6822, and the Sagittarius irregular and dwarf elliptical galaxies) that lie within $10^{\circ}$ of the projected orbits. However, with the exception of the Sagittarius dwarf elliptical (dE), their measured distances are all greater than $500 \mathrm{kpc}$ and would therefore require rather unreasonably high orbital eccentricities $(\epsilon>0.98)$. We attribute the proximity of the Sagittarius $\mathrm{dE}$ to the new stream's projected orbit to the expected confluence of orbit projections in the direction of the Galactic center and not to any physical association between them.

In the upper right-hand corner of Figure 1 (R.A., decl.) = $\left(132^{\circ} .857,+63^{\circ} .136\right)$ is a fairly pronounced concentration of stars about $37 \mathrm{kpc}$ distant that we identify as a new globular cluster or dwarf galaxy (C. J. Grillmair 2006, in preparation). The object lies within $5^{\circ}$ of the projected orbit of the stream $(1.8 \mathrm{kpc}$ at the distance of the stream). However, its diminutive size and its $16 \mathrm{kpc}$ separation from the stream's orbit argue against it being either the progenitor or a part of the debris in the stream.

\section{CONCLUSIONS}

Applying optimal contrast filtering techniques to SDSS data, we have detected a broad stream of stars some $60^{\circ}$ long on the sky. We are at present unable to identify a progenitor for this stream, although from its appearance and location on the sky, we believe it to be a either an extant or disrupted dwarf galaxy. The color-magnitude distribution of stars in the stream closely matches that of the globular cluster M13, indicating that the stars making up the stream are old and metal-poor. The stream appears to be about $21 \mathrm{kpc}$ distant, and the portion of the stream contained in the survey data is roughly perpendicular to our line of sight.

Refinement of the stream's orbit will require radial velocity measurements of individual stars along its length. Ultimately, the vetted stream stars will become prime targets for the Space Interferometry Mission, whose proper-motion measurements will enable very much stronger constraints to be placed on both the orbit of the progenitor and the potential field of the Galaxy.

Funding for the creation and distribution of the SDSS Archive has been provided by the Alfred P. Sloan Foundation, the Participating Institutions, the National Aeronautics and Space Administration, the National Science Foundation, the US Department of Energy, the Japanese Monbukagakusho, and the Max Planck Society.

Facilities: Sloan

\section{REFERENCES}

Allen, C., \& Santillan, A. 1991, Rev. Mex. AA, 22, 255

Bellazzini, M., Gennari, N., Ferraro, F. R., \& Sollima, A. 2004, MNRAS, 354, 708

Belokurov, V., Evans, N. W., Irwin, M. J., Hewett, P. C., \& Wilkinson, M. I. 2006a, ApJ, 637, L29

Belokurov, V., et al. 2006b, ApJ, 642, L137

Dolphin, A. E., et al. 2002, AJ, 123, 3154

Grillmair, C. J., \& Dionatos, O. 2006a, ApJ, 641, L37 2006b, ApJ, 643, L17

Grillmair, C. J., \& Johnson, R. 2006, ApJ, 639, L17

Majewski, S. R., Skrutskie, M. F., Weinberg, M. D., \& Ostheimer, J. C. 2003, ApJ, 599, 1082
Martinez-Delgado, D., Gomez-Flechoso, M., Aparicio, A., \& Carrera, R. 2004, ApJ, 601, 242

Mateo, M., Fischer, P., \& Krzeminski, W. 1995, AJ, 110, 2166

Odenkirchen, M., et al. 2001, ApJ, 548, L165 2003, AJ, 126, 2385

Rocha-Pinto, H. J., Majewski, S. R., Skrutskie, M. F., Crane, J. D., \& Patterson, R. J. 2004, ApJ, 615, 732

Rockosi, C. M., et al. 2002, AJ, 124, 349

Schlegel, D. J., Finkbeiner, D. P., \& Davis, M. 1998, ApJ, 500, 525

Willman, B., et al. 2005, AJ, 129, 2692

Yanny, B., et al. 2003, ApJ, 588, 824

Zucker, D. B., et al. 2006, ApJ, 643, L103 